\title{
ORIGINAL
}

\section{Our experience of treatment of cribriform morular variant of papillary thyroid carcinoma; difference in clinicopathological features of FAP-associated and sporadic patients}

\author{
Yasuhiro Ito $^{1)}$, Akira Miyauchi ${ }^{1)}$, Hideki Ishikawa ${ }^{2)}$, Mitsuhiro Hirokawa ${ }^{3)}$, Takumi Kudo ${ }^{4)}$, Chisato Tomoda ${ }^{1)}$ \\ and Akihiro Miya ${ }^{1)}$ \\ 1) Department of Surgery, Kuma Hospital, Kobe, Japan \\ ${ }^{2)}$ Department of Molecular-Targeting Cancer Prevention, Graduate School of Medical Science, Kyoto Prefectural University of \\ Medicine, Kyoto, Japan \\ 3) Department of Pathology, Kuma Hospital, Kobe, Japan \\ 4) Department of Internal Medicine, Kuma Hospital, Kobe, Japan
}

\begin{abstract}
Cribriform-morular variant (CMV) is a comparably rare histological subtype of papillary thyroid carcinoma (PTC). This can be associated with familial adenomatous polyposis (FAP) due to APC gene mutations. In this study, we investigated the difference in the biological characteristics between FAP-associated and sporadic CMV. Between 1991 and 2010, 32 patients with CMV were treated in Kuma Hospital. Thirty-one of these underwent initial surgery for CMV in Kuma Hospital. Twelve patients were FAP-associated and the remaining 19 were sporadic CMV. All patients were female. Tumors of FAP-associated CMV were more frequently multiple than those of sporadic CMV. Patient age and tumor size did not differ between the two groups. Of 12 FAP-associated CMV, 5 were detected by thyroid nodule (thyroid precedent group) and 7 were detected by FAP (polyposis precedent group) as an initial manifestation. Patient age was younger and tumor size was smaller in the polyposis group than in the thyroid nodule group. All patients lacked extrathyroid extension on intraoperative finding and were node-negative on pathological examination. To date, two patients with FAP-associated CMV who initially underwent hemithyroidectomy (one in Kuma Hospital and one in another hospital) showed recurrence to the remnant thyroid during follow-up. None of the patients showed recurrence to other regions or died of carcinoma. Taken together, CMV is considered an indolent disease in our series. FAP-associated CMV showed multiple tumors more frequently than sporadic CMV. Total thyroidectomy is recommended for FAP-associated CMV, but extensive lymph node dissection is not necessary.
\end{abstract}

Key words: Cribriform morular variant, Papillary thyroid carcinoma, FAP, Prognosis

PAPILLARY thyroid carcinoma (PTC) is the most common malignancy arising from thyroid follicular cells. There are many histological subtypes of PTC and some of them showed a different prognosis from conventional PTC. Cribriform-morular variant (CMV) is a rare subtype of PTC. Historically, in 1949, Crail first reported malignancies originating from the rectum, brain and the thyroid gland [1]. In 1968, the relation between familial adenomatous polyposis (FAP) and thyroid carcinoma was reported [2]. Furthermore, one

Received May 12, 2011; Accepted May 30, 2011 as EJ11-0022 Released online in J-STAGE as advance publication Jun. 14, 2011

Correspondence to: Yasuhiro Ito, M.D., Ph.D. Department of Surgery, Kuma Hospital, 8-2-35, Shimoyamate-dori, Chuo-ku, Kobe 650-0011, Japan. E-mail: ito01@kuma-h.or.jp study showed that young women with FAP had approximately 160 times more risk of thyroid carcinoma than healthy people [3]. In 1994, Harach et al. demonstrated unique histological features of FAP-associated PTC such as a cribriform pattern and solid areas with a spindle cell component [4]. The APC gene located in the 5q21 region is strongly associated with FAP and individuals with $A P C$ mutations have almost $100 \%$ risk of developing colorectal carcinoma [5, 6], allowing us to conclude that this variant is significantly associated with $A P C$ mutations. Other studies have also reported FAP-associated CMV [7, 8], but CMV can occur also sporadically without any relation to colonic polyposis [8-10].

In 2004, we reported 7 patients with CMV [11]. Three 
of these patients were FAP-associated CMV because they had FAP or a family history of colonic carcinoma involving $A P C$ gene mutations. The remaining 4 were sporadic and lacked APC gene mutations. However, primary lesions in all of these patients were detected by thyroid nodule as an initial manifestation. Recently, the relationship between CMV and FAP has been more widely understood and CMV has been detected during thyroid screening in FAP patients without complaint of thyroid nodule.

In this study, we investigated the biological characteristics of CMV in 32 patients. Thirty-one patients underwent initial surgery in Kuma Hospital. Twenty of these patients were FAP-associated and the remaining 19 were sporadic CMV. We investigated the difference in clinicopathological features between these two groups.

\section{Patients and Methods}

Between 1991 and 2010, 32 patients with PTC underwent surgery and pathologically diagnosed as CMV in Kuma Hospital, who were enrolled in this study. All patients were females and patient age ranged from 17 to 38 years (average 27 years). These 32 patients underwent ultrasound-guided fine needle aspiration biopsy (FNAB) and were cytologically diagnosed as having PTC. Sixteen (50\%) were also diagnosed as or highly suspected of CMV based on cytological findings such as the presence of a morular component, peculiar nuclear clearing, absence of colloid in the background and immunocytochemistry finding such as nuclear and cytoplasmic localization of beta-catenin and high positivity of estrogen receptor (ER) $[12,13]$.

Thirty-one of these patients underwent initial surgery in Kuma Hospital. Twelve of these patients (31\%) were FAP-associated CMV because they had one or more of the following three features; 1) colonic polyposis was detected on total colonoscopy before or after thyroid surgery; 2) presence of APC gene mutations; and 3) family history of colonic polyposis or colorectal carcinoma. CMV in 5 patients was detected by thyroid nodule based on patient complaints or findings on medical check-up as the initial event. These patients underwent total colonoscopy after thyroid surgery and were diagnosed as having polyposis. The remaining 7 underwent thyroid ultrasound for screening after the detection of FAP and were referred to our hospital because thyroid nodules were found. None of these patients had complained of thyroid nodule prior to its detection. Nineteen patients (69\%) were diagnosed as having sporadic CMV without any of the 3 features, which includes 3 patients who had no family history of colonic polyposis or colorectal carcinoma and rejected examination of colonic polyposis.

The extent of thyroidectomy for FAP-associated CMV was total thyroidectomy in 11 patients and hemithyroidectomy in 1 patient. That for sporadic CMV was total thyroidectomy in 9 patients and hemithyroidectomy in 10 patients. Completion total thyroidectomy was performed as the second surgery in one patient who underwent initial surgery at another hospital. All patients underwent central node dissection. Three patients with FAP-associated CMV and 8 with sporadic CMV also underwent modified radical neck dissection (MND). All patients were diagnosed as having CMV on postoperative pathological examination based on morphological findings such as cribriform pattern, morular component, lack of colloid, peculiar nuclear clearing and immunohistochemical findings of beta-catenin, ER and progesterone receptor (PgR) $[4,8,9,14]$.

Our series included 7 patients reported in our previous study who underwent $A P C$ gene mutation analysis [11]. The APC gene mutation was not analyzed in other patients.

None of the patients in our series underwent radioactive iodine (RAI) ablation or RAI therapy. After surgery, all patients were followed by ultrasound at least once per year. The follow-up periods of these 31 patients ranged from 4 to 217 months (average 59 months).

Fisher's exact test and Mann-Whitney U test were adopted for comparing variables. $p$ values less than 0.05 were considered significant.

\section{Results}

Of 32 patients with CMV, 31 underwent initial surgery in Kuma Hospital. Twelve of these patients (39\%) were diagnosed as having FAP-associated CMV because they had one or more of the following three features as indicate above, and the remaining 19 (61\%) demonstrated sporadic CMV. Nine patients with FAP-associated CMV (75\%) and 7 with sporadic CMV (37\%) were preoperatively diagnosed as having or highly suspected of CMV based on the cytological findings indicated above. Multinodular goiter and euthyroid Graves' disease were detected in 3 patients and 1 patient with sporadic CMV, respectively. No 
Table 1 Comparison of multiplicity on ultrasound for FAPassociated and sporadic CMV

\begin{tabular}{lccc}
\hline & \multicolumn{3}{c}{ Multilplicity (\%) } \\
\cline { 2 - 4 } & Solitary & Multiple & Total \\
\hline FAP-associated CMV & $3(25)$ & $9(75)$ & 12 \\
Sporadic CMV & $19(100)$ & 0 & 19 \\
Total & 22 & 9 & 31 \\
\hline
\end{tabular}

$p=0.00001$
Table 2 Comparison of multiplicity on pathological examination for FAP-associated and sporadic CMV

\begin{tabular}{lccc}
\hline & \multicolumn{3}{c}{ Multilplicity (\%) } \\
\cline { 2 - 4 } & Solitary & Multiple & Total \\
\hline FAP-associated CMV & $2(17)$ & $10(83)$ & 12 \\
Sporadic CMV & $17(89)$ & $2(11)$ & 19 \\
Total & 19 & 12 & 31 \\
\hline
\end{tabular}

$p=0.0005$

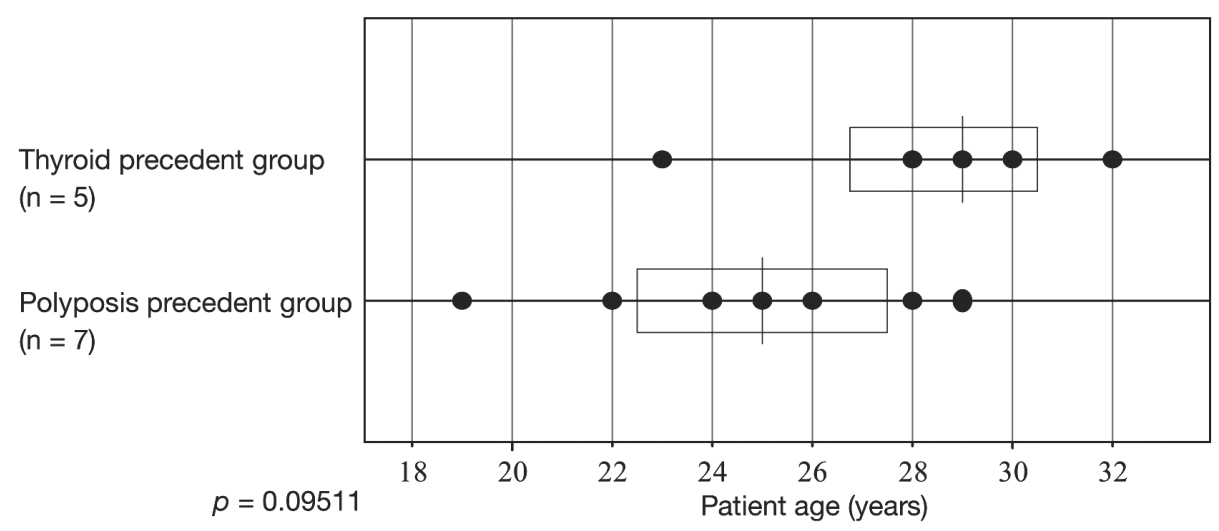

Fig. 1 Comparison of age of FAP- associated patients between the thyroid precedent group and polyposis precedent group.

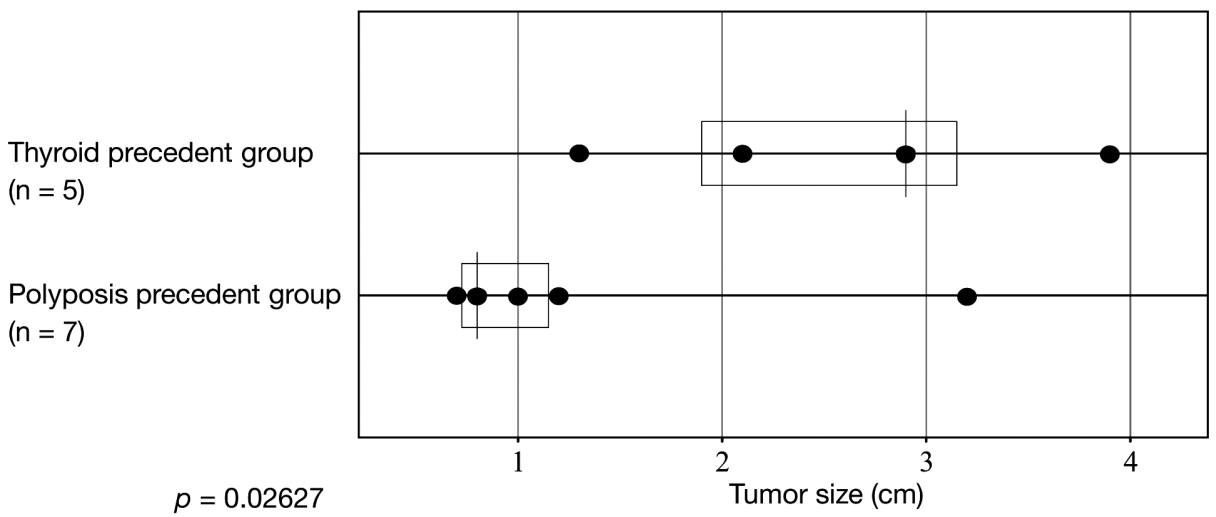

Fig. 2 Comparison of tumor size of FAP- associated patients between the thyroid precedent group and polyposis precedent group.

other thyroid comorbidities can be observed in FAPassociated CMV.

We compared the clinicopathological features between the two groups. Tumor size $(1.79+1.15 \mathrm{~cm}$ vs $2.38+0.70 \mathrm{~cm})$ and patient age $(26.3+3.7$ years $v$ s $26.8+6.6$ years) did not significantly differ between FAP-associated and sporadic CMV. Nine of 12 patients (75\%) with FAP-associated CMV but none of the sporadic CMV were evaluated as multiple on preoperative ultrasound ( $p=0.00001)$ (Table 1). On pathological examination, 10 patients with FAP-associated CMV (83\%) and 2 with sporadic CMV (11\%) showed multi- plicity $(p=0.00005)$ (Table 2$)$.

Diagnosis of CMV in 5 of 12 FAP-associated CMV patients $(42 \%)$ was based on finding a thyroid nodule (thyroid precedent group) and that in 7 (58\%) was based on FAP (polyposis precedent group) as the initial manifestation. Although not significantly different, patient age in the thyroid precedent group $(28.4+3.4$ years $)$ tended to be older than that in the polyposis precedent group $(24.7+3.5$ years $)(p=0.09511)$ (Fig. 1$)$. Tumor size in the thyroid precedent group $(2.6+1.0 \mathrm{~cm})$ was significantly larger than that in the polyposis precedent group $(1.2+0.9 \mathrm{~cm})(p=0.02627)$ (Fig. 2). 
None of the patients showed extrathyroid extension corresponding to $\mathrm{T} 3$ or $\mathrm{T} 4$ or lymph node metastasis corresponding to N1 in the UICC TNM classification [13] based on preoperative and intraoperative findings. On pathological examination, all patients were nodenegative. To date, two patients with FAP-associated CMV, who underwent hemithyroidectomy at the initial surgery (one in Kuma hospital and one in another hospital) and were diagnosed as having polyposis thereafter, showed recurrence to the remnant thyroid 86 and 90 months after initial surgery, respectively. These patients underwent a second surgery and further recurrence has not been detected to date. Furthermore, one patient died of colorectal carcinoma 64 months after thyroid surgery and one died of lung suppuration unrelated to PTC or colorectal carcinoma 28 months after surgery. The other patients have survived without any evidence of PTC recurrence to date.

\section{Discussion}

There are two types of CMV etiology; FAPassociated and sporadic. Furthermore, there are two initial manifestations of FAP-associated CMV detection: thyroid nodule and FAP. We compared clinicopathological features between the two groups.

CMV can be diagnosed by preoperative cytological findings [12]. In our series, $75 \%$ of FAP-associated CMV and $37 \%$ of sporadic CMV were diagnosed as or highly suspected of CMV on cytology. The higher incidence in FAP-associated CMV is definitely because we ordered immunocytochemistry for beta-catenin and ER for patients who were known to have FAP.

Since it is well-known that CMV can be associated with FAP, screening of colonic polyposis by total colonoscopy should be recommended for patients who were diagnosed as having CMV. We demonstrated that FAP-associated CMV showed multiple tumors more frequently than sporadic CMV both on preoperative imaging studies and on pathological examination, indicating that the presence of FAP is highly suspected especially for patients with multiple CMV. In the subset of FAP-associated CMV, patients in the polyposis precedent group were younger and had smaller tumors than those in the thyroid precedent group. It is therefore suggested that thyroid screening for FAP patients contributes to early detection of CMV, even before patients become aware of thyroid nodule.

All patients in our series were node-negative and lacked extrathyroid extension. Furthermore, none of these patients died of carcinoma. These findings indicate that CMV normally shows an indolent character regardless of whether it is FAP-associated, which was not discrepant with previous studies $[8,9,10,16]$. There is one report of CMV in an older (42 years) male patient who died of carcinoma only 17 months after surgery, but this seems atypical because neuroendocrine differentiation and a poorly differentiated component were present [17]. To date, however, 2 patients who underwent hemithyroidectomy for FAP-associated CMV recurred to the remnant thyroid during follow-up. Based on these findings, total thyroidectomy is recommended for CMV patients with multiple carcinoma lesions, a family history of colonic polyposis or colorectal carcinoma or diagnosed as having colonic polyposis before thyroid surgery. In contrast, none of the sporadic patients showed recurrence to the remnant thyroid, even though they underwent hemithyroidectomy, indicating that total thyroidectomy may not be mandatory for such patients. In our department, prophylactic MND has been performed for PTC patients having tumor larger than $3 \mathrm{~cm}$, because these patients are likely to develop recurrence in regional lymph nodes [18]. However, such an extensive prophylactic node dissection is not needed for patients who were preoperatively diagnosed as having CMV.

Taken together, we showed that FAP-associated CMV showed multiple tumors more frequently than sporadic CMV. Total thyroidectomy is recommended at least for CMV patients associated with FAP, but extensive lymph node dissection is not necessary.

\section{References}

1. Crail HW (1949) Multiple primary malignancies arising in the rectum, brain, and the thyroid: report of a case. $U$ S Nav Med Bull 49: 123-128.

2. Camiel MR, Mule JE, Alexander LL, Benninghoff DL
(1968) Association of thyroid carcinoma with Gardner's syndrome in siblings. N Engl J Med 278: 1056-1058.

3. Plail RO, Bussey HJ, Glazer G, Thomspon JP (1987) Adenomatous polyposis: an association with carcinoma 
of the thyroid. Br J Surg 74: 377-380.

4. Harach HR, Williams GT, Williams ED (1994) Familial adenomatous polyposis associated thyroid carcinoma: a distinct type of follicular cell neoplasm. Histopathology 25: 549-561.

5. Groden J, Thliveris A, Samowitz W, Carlson M, Gelbert L, Albertsen H, Joslyn G, Stevens J, Spiro L, Robertson M (1991) Identification and characterization of the familial adenomatous polyposis coli. Gene. Cell 66: 589-600.

6. Kinzler KW, Nibert MC, Su LK, Vogelstein B, Bryan TM, Levy DB, Smith KJ, Preisinger AC, Hedge P, McKechnie D (1991) Identification of FAP locus gene from chromosome 5q21. Science 253: 661-665.

7. Cetta F, Pelizzo MR, Caria MC, Barbarisi A (1999) Genetics and clinicopathological findings in thyroid carcinomas associated with familial adenomatous polyposis. Am J Pathol 155: 7-9.

8. Cameselle-Teijeiro J, Chan JK (1999) Cribriformmorular variant of papillary carcinoma: A distinctive variant representing the sporadic counterpart of familial adenomatous polyposis-associated thyroid carcinoma? Mod Pathol 12: 400-411.

9. Ng SB, Sittampalm K, Goh YH, Eu KW (2003) Cribriform-morular variant of papillary carcinoma: The sporadic counterpart of familial adenomatous polyposis-associated thyroid carcinoma. A case report with clinical and molecular genetic correlation. Pathology 35: 42-46.

10. Xu B, Yoshimoto K, Miyauchi A, Kuma S, Mizusawa N, Hirokawa M, Sano T (2003) Cribirform-morular variant of papillary thyroid carcinoma: A pathological and molecular genetic study with evidence of frequent somatic mutations in exon 3 of the beta-catenin gene. $J$ Pathol 199: 58-67.

11. Tomoda C, Miyauchi A, Uruno T, Takamura Y, Ito Y, Kobayashi K, Matsuzuka F, Kuma S, Kuma K, Kakudo K (2004) Cribriform-morular variant of papillary thy- roid carcinoma: clue to early detection of familial adenomatous polyposis-associated colon cancer. World $J$ Surg 28: 886-889.

12. Hirokawa M, Maekawa M, Kuma S, Miyauchi A(2010) Cribriform-morular variant of papillary thyroid carcinoma-cytological and immunocytochemical findings of 18 cases. Diag Cytopathol 38: 890-896.

13. Kuma S, HIrokawa M, Xu B, Miyauchi A, Kakudo K, Sano T (2004) Cribriform-morular variant of papillary thyroid carcinoma. Report of a case showing morules with peculiar nuclear clearing. Acta Cytol 48: 431436.

14. Kurihara K, Shimizu S, Chong J, Hishima T, Funata N, Kashiwagi H, Nagai H, Miyaki M, Fukayama M (2000) Nuclear localization of immunoreactive beta-catenin is specific to familial adenomatous polyposis in papillary thyroid carcinoma. Jpn J Cancer Res 91: 1100-1102.

15. Sobin LH, Wittekind Ch., eds. (2002) UICC; TNM classification of malignant tumors, $6^{\text {th }}$ ed. New York: WileyLiss.

16. Perrier ND, van Heerden JA, Goellner JR, Williams ED, Gharib H, Marchesa P, Church JM, Fazio VW, Larson DR (1998) Thyroid cancer in patients with familial adenomatous polyposis. World J Surg 22: 738-742.

17. Cameselle-Teijeiro J, Menasce LP, Yap BK, Colaco RJ, Castro P, Celestino R, Ruiz-Ponte C, Soares P, SobrinhoSimoes M (2009) Cribriform-morular variant of papillary thyroid carcinoma. Molecular characterization of a case with neuroendocrine differentiation and aggressive behavior. Am J Clin Pathol 131: 134-142.

18. Ito Y, Higashiyama T, Takamura Y, Miya A, Kobayashi K, Matsuzuka F, Kuma K, Miyauchi A (2007) Risk factors for recurrence to the lymph node in papillary thyroid carcinoma patients without preoperatively detectable lateral node metastasis: validity of prophylactic modified radical neck dissection. World J Surg 31: 20852091. 